\title{
Integrated modelling of contemporaneous multi-utility demand data from intelligent meters
}

\author{
$\underline{\text { K.A. Nguyen }}^{\text {a }}$, R.A. Stewart ${ }^{\text {a }}$, H. Zhang $^{\text {a }}$ and O. Sahin $^{\text {a }}$ \\ ${ }^{a}$ Griffith School of Engineering, Cities Research Institute, Griffith University, Queensland, Australia \\ Email: k.nguyen@griffith.edu.au
}

\begin{abstract}
With the advent of smart metering in recent years where water and energy consumption data could be recorded at high resolution, several studies have been undertaken all over the world to unpack various benefits for both consumers and suppliers. Separate analysis and modelling of water or energy data has shown valuable state-of-the-art applications to inform single and multi-utilities and regulatory agencies. This paper suggests a new concept where high resolution multi-utility data is concurrently collected and modelled to allow for enhanced pattern recognition of other resources (e.g. having electricity data assists pattern recognition of water), deeper insight into customer demand and optimal opportunities to manage it. Through using a smart device to capture concurrent water and energy consumption in near real-time, and exploring the correlation between these two consumption activities, the proposed system has helped avoid the need of using Hidden Markov Model and Dynamic Time Warping algorithms in several analysis stages, thus allowed the classification process to be undertaken much faster with higher achieved accuracy. Once finished, the system will result in a wide range of benefits for utilities and regulatory agencies, especially allowing them to have a unique single platform to monitor all water and energy consumption of any particular household or region in near real-time to immediately identify faulty issue with the power system or pipe leakage if it happens. For customer, they will also be immediately alerted when there is any single problem occurring to any water or energy device, or be informed about the current efficiency status of water and power appliance in the house, as well as receiving various incentives when they follow instruction to improve the current supply network.
\end{abstract}

Keywords: Water consumption, energy consumption, end use 


\section{INTRODUCTION}

The coupled analysis and modelling of water and energy data has shown valuable state-of-the-art applications to inform single and multi-utilities and regulatory agencies. Indeed, low and medium resolution data can be exploited to perform urban scale studies aimed at assessing the environmental impacts and costs of waterrelated energy (Escriva-Bou et al. 2015), as well as exploring heterogeneous consumption patterns. In contrast, detailed end-use water consumption data requires higher resolution digital water meters, capable of measuring very low flow rates (e.g. $0.01 \mathrm{~L}$ ) at close logging intervals (e.g. 5s) (Giurco et al. 2008a). In the energy industry where smart electricity and gas meters and communications infrastructure have already been more widely introduced, low-medium power consumption data collected at minute or hourly interval have been effectively used in power demand forecasting, or design of customized energy demand management strategies. Several algorithms for high-resolution power consumption end-use disaggregation have been proposed and summarised in a review paper by (Zoha et al. 2012). It is also noted that, to the authors knowledge, no study was conducted so far to utilise high resolution concurrent signal to improve the achieved accuracy of this disaggregation process, which potentially open a new space in this research area.

\section{BACKGROUND}

High resolution water-energy data contain a wide spectrum of useful information which, if thoroughly explored, would allow utilities to achieve much more effective management schemes. One of the most notable uses of concurrent data is in assisting the task of disaggregating power and water consumption data into different end-use categories. Some certain appliances in a household require both water and energy to function, such as clothes washer and dishwasher, and the overlapping of water and energy patterns would allow the classification of these end-uses to be achieved almost at the absolute accuracy. In water end-use disaggregation, the misclassification usually occurred due to the pattern similarity of different categories. A hybrid combination of Hidden Markov Model, Dynamic Time Warping Algorithm and Artificial Neural Network has usually been applied to address this issue (Nguyen et al., 2015), however, the overall process is relatively computationally expensive.

With the need for further improved classification accuracy and reduced computation expense for this water end-use classification problem, this paper advocates a vision of a multi-utility where demand data is concurrently collected and modelled to allow for enhanced pattern recognition mechanism (e.g. having electricity data assists pattern recognition of water), deeper insight into customer demand and strategies to manage it, as well better water and electricity grid infrastructure asset management. A demonstration of how the classification of end-uses can benefit both joint water and energy data is described here through the classification of clothes washer use events using concurrent water-energy signals, and comparing the outcomes against those obtained from separate water or energy data.

\section{METHOD}

\subsection{End-use classification using separate signal}

In terms of energy, smart meter data collected at minute or hourly interval was used as the main resource, and several techniques have been applied to achieve this task, including genetic algorithms, integer optimization, sparse optimization, Factorial Hidden Markov Model, Dynamic Time Warping Algorithm, or Hybrid Signature-Based Iterative Disaggregation algorithm (Cominola, 2017). In water domain, range of recorded accuracy from $80 \%-94 \%$ was reported in (Nguyen et al., 2014) when several techniques including Hidden Markov Model, Dynamic Time Warping Algorithm, Probabilistic Model and Artificial Neural Network were all combined to analyse smart water data collected at 5 seconds interval with the resolution of 72 pulse per litre. To further improve these achieved accuracies, the use of concurrent water-energy signal was proposed.

\subsection{Building a power consumption data collection toolkit}

To collect water consumption data at 5 second interval, commercial Aquiba smart water meter was used in the study. In terms of energy consumption, as no commercial product available on the market allows 5 -second data to be collected and downloaded for analysis, a special toolkit was developed by the author to achieve this purpose.

Presented in Figure 1a is a current transformer (CT) that is used to produce an alternating current (AC). This CT is clipped over the live power supply cable in the household meter box. A microcontroller (left hand side 
device) is then connected to this CT via a $3.5 \mathrm{~mm}$ jack, and is programmed to send power consumption data from $\mathrm{CT}$ to a raspberry pi device every five seconds, which is already programed to receive, decode and display the power consumption in real-time. Data collected from smart water meter is also captured using a script written in Python language and is displayed on the Raspberry Pi device concurrently with energy (Figure 1b).

The collected concurrent data is presented in Figure 3 below which demonstrates how the water (red) and energy signals (blue) are overlaid so that patterns can be detected.

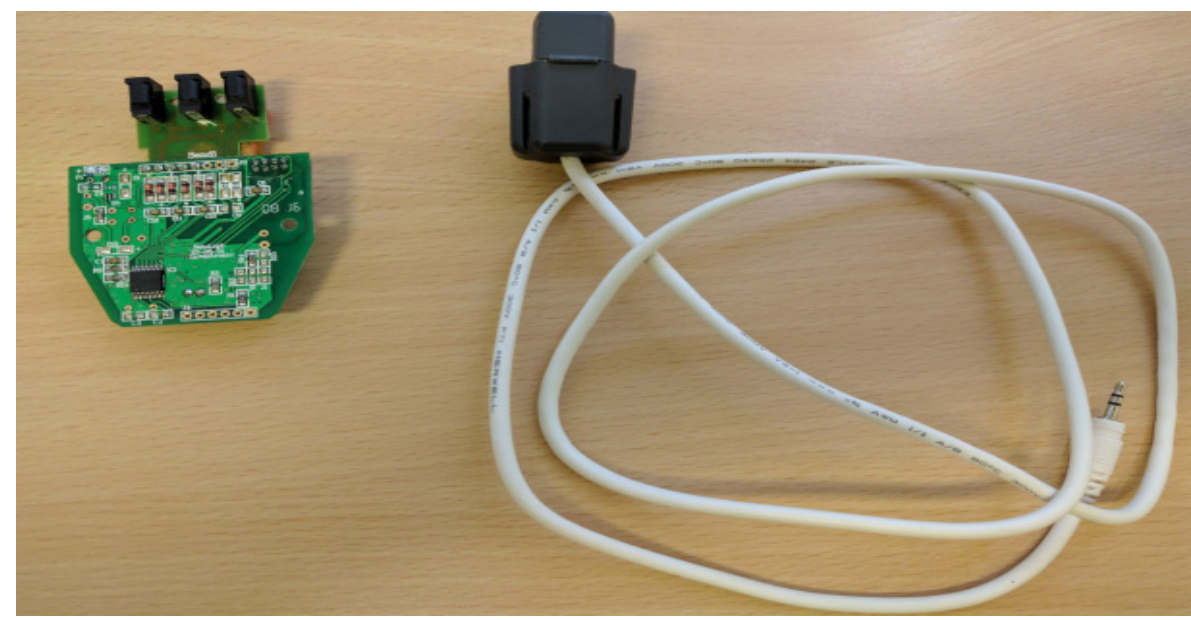

a. Current transformer and Arduino board

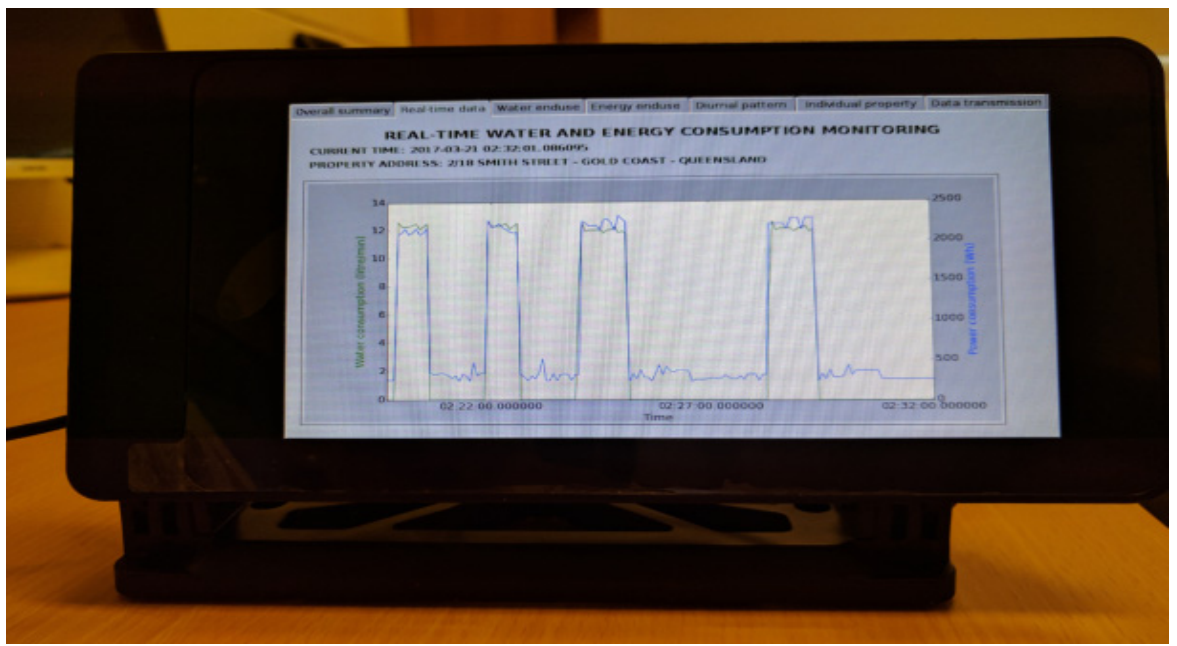

b. Raspberry pi with touchscreen

Figure 1. Power consumption data collection toolkit

\subsection{End-use classification using concurrent signal}

Through collecting both concurrent water and energy consumption at the same time, this study has proposed a smart system that allows both water and energy consumption to be disaggregated into end-use level in almost real-time. The water end-use disaggregation techniques using concurrent data is presented through an illustrative example in Section 4. With energy disaggregation, the concurrent data has allowed clothes washer, dishwasher, hot water to be isolated. Kalman Filter was then applied to identify power events that last over a long period of time (e.g. fridge and lights). For other events that have repeated pattern such as air conditioner and microwave, Dynamic Time Warping algorithm was used as the main classification technique. Since energy 
disaggregation is not the main goal of this paper, a detailed description of this process will be published in other occasion. Once the whole water-energy end-use analysis was accomplished, two consumption pie charts were presented for one of the trial properties in the study (Figure 2).

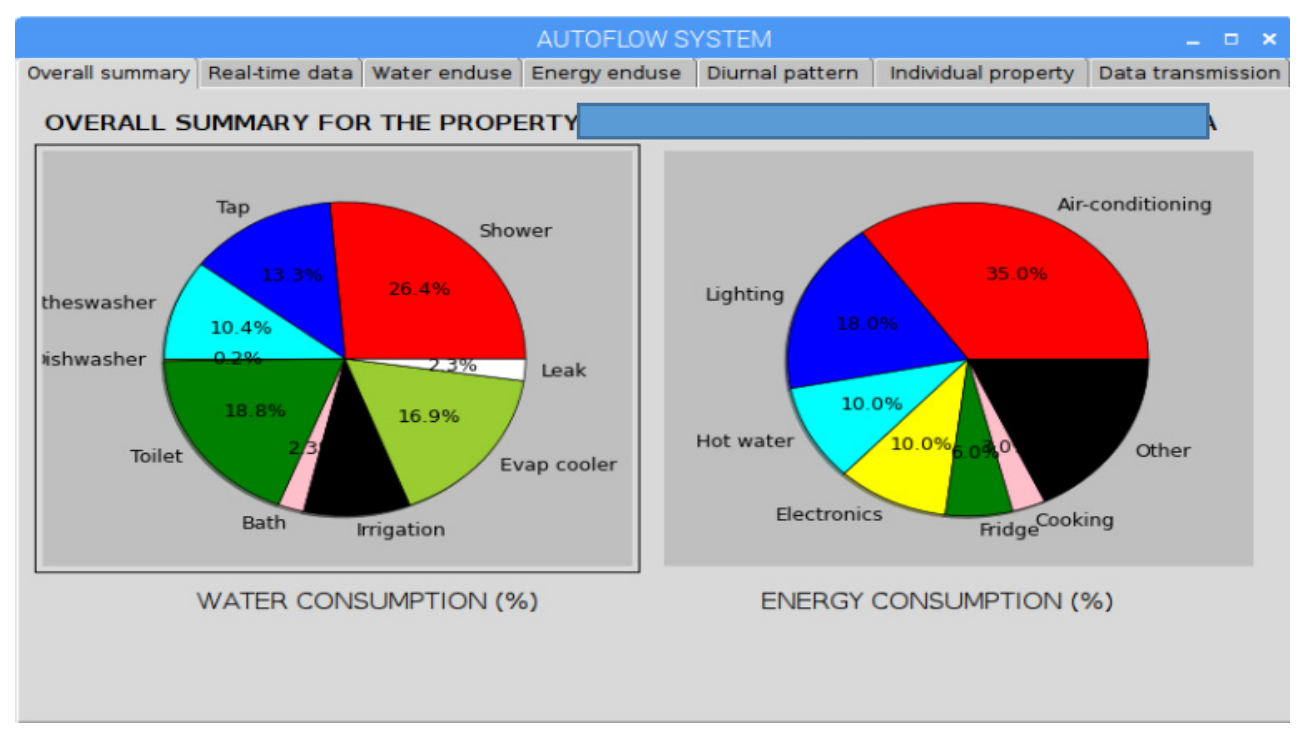

Figure 2. Overall summary of water and energy end use disaggregation

\section{ILLUSTRATIVE EXAMPLE WITH CLOTHESWASHER EVENT CLASSIFICATION}

Classification of clothes washer event is used to illustrate the benefits of a concurrent method. Clothes washer is one of the essential appliances present in every household, and the classification of end-uses like this is a major part of all studies relating to water and energy consumption disaggregation (Nguyen et al., 2015; Anderson, 2014).

In conventional practice, the misclassification of clothes washer event in water end use disaggregation task usually occurred due to the similarity of its pattern with toilet or tap. The application of Dynamic Time Warping algorithm to find repeated pattern (i.e. as one clothes washer load contains several similar cycle) is the best option so far to largely address this problem, however, the computation when using this technique is very expensive, especially when dealing with a large data set. Moreover, the achieved accuracy is still around 90$94 \%$ as reported in (Nguyen et al, 2014) when using water data alone. Once the concurrent power consumption is also recorded, the analysis time for this water end-use disaggregation process can be reduced dramatically while the accuracy can be boosted to almost around $98 \%$. Moreover, the direct advantage of increased disaggregation capabilities for those end-uses that use both water and energy will reflect in an overall improvement of the disaggregation process, and, in turn, more accurate disaggregation of those end-uses that do not use energy (e.g., toilet flush) or water (e.g., clothes dryer).

For water end-use classification, the collected concurrent data (Figure 3) shows a strong correlation of water (red) and energy signal (blue) when the two patterns are overlaid. More specifically, it indicates that during spinning process, water is injected while power is used to spin the drum, which resulted in two perfectly matching signals. It is this special feature which would significantly enhance the classification of clothes washer event. 


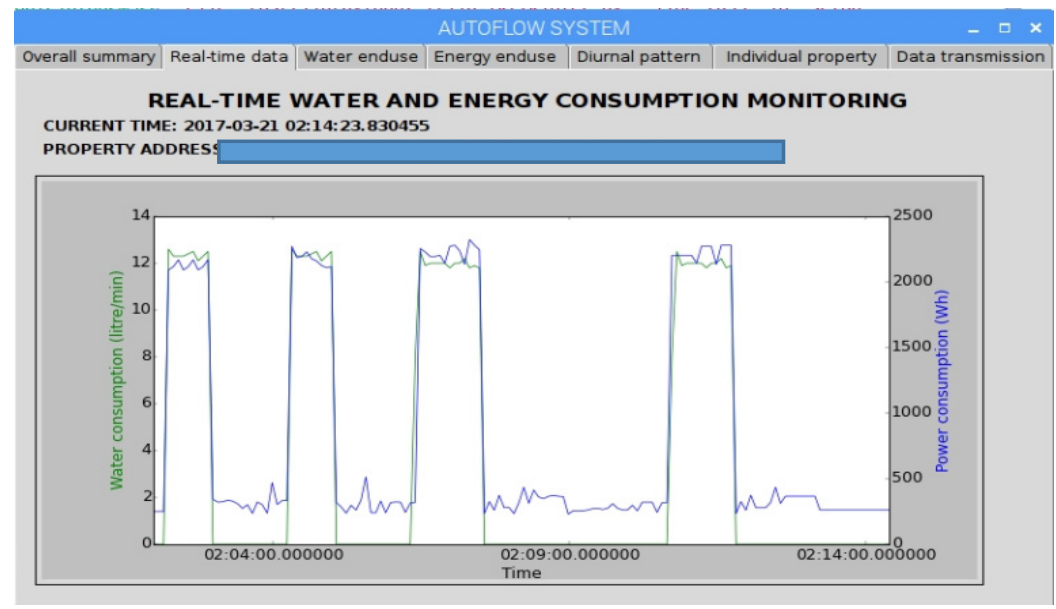

Figure 3. Concurrent power-water signal collected every 5 seconds

The very first step of the overall process is to overlay these two signals (i.e. water and energy) and screen out all events that concurrently occurred. This process would allow all clothes washer and dishwasher (if there is as dishwasher it also operates similarly) to be separated from the remaining events. As dishwasher water events have much lower flow rate compared to clothes washer, these two end uses could be immediately separated as well. This process would avoid the need of using Hidden Markov Model and Dynamic Time Warping algorithm for clothes washer and dishwasher classification, which allows the analysis to be completed several times faster. Apart from these two end use categories, the concurrent signal would also improve the accuracy for shower and bathtub classification in some cases as these two also use hot water which requires the draw of power for heating. Moreover, if water is heated by gas, the availability of gas usage data would complement those of water and electricity, contributing to further improvements on end-use disaggregation performance.

\section{APPLICATIONS}

\subsection{Application for utilities and regulatory agencies}

The collection and analysis of concurrent water and energy data will result in a wide range of benefits for utilities and regulatory agencies. This includes: (1) having a unique single platform to monitor all water and energy consumption of any particular household or region in near real-time to immediately identify issues (e.g. household water leaks); (2) optimised water and energy grid infrastructure asset management; (3) improved customer satisfaction when user is provided with comprehensive information regarding the efficiency of their appliances (e.g. "your clothes washer water and energy consumption per load are $140 \mathrm{~L}$ and $1300 \mathrm{~W}$, which is much higher than the average consumption of $70 \mathrm{~L}$ and $800 \mathrm{~W}$ '); and (4) provides utilities and regulators to develop more effective demand management messaging and strategies (e.g. rebates, education, restrictions, etc.) during periods of water scarcity or peak energy periods by using information derived from the concurrent data.

\subsection{Application for customers}

Customers will gain significant benefits from the analysis and presentation of concurrent water-energy data from a multi-utility provider, including: (1) one single account to view both real-time water and energy consumption as well as other statistical reports, including comparisons with other households with similar demographic patterns, notification from suppliers, detailed end use disaggregation, or recommendations to help reduce consumption; (2) be immediately alerted when water or energy demand is uncharacteristic (e.g. water leak in home); and (3) be informed about the current efficiency status of water and power appliances and devices; (4) receipt of rewards when shifting or reducing demand on the request of the utility to achieve certain supply or distribution grid infrastructure management objectives (e.g. Message from utility: "Please avoid using clothes washer and dishwasher between 6 to 8pm tomorrow. In return, you will receive ten reward points to your account. Once 50 points has been accumulated, you will receive a $\$ 10$ discount on your next bill"). 


\section{CONCLUSION}

The realisation that residential water and energy consumption data are related has prompted researchers to analyse collected data to reveal efficiency opportunities (Beal et al. 2012; Beal et al. 2016). Moreover, while there is some anecdotal understanding of the relationship between customer water and energy demand, there is still a lack of evidence-based research supporting certain water-energy nexus trends. Detailed understanding has not been possible because until recently there has been limited high resolution water and energy data available, especially concurrently occurring energy (electricity and/or gas) and water data that can be directly correlated to reveal interesting water-energy nexus trends. This paper addresses this need by describing a tool to generate datasets, and illustrating its use in a case study that showed how this tool and concurrent analysis could a) reduce computational demand, and b) improve the accuracy of disaggregation

\section{REFERENCE}

Anderson, K (2014) Non-Intrusive Load Monitoring: Disaggregation of Energy by Unsupervised Power Consumption Clustering - Doctor of Philosophy thesis - Carnegie Merlon University

Beal, C. D., Stewart, R.A., \& Bertone, E. (2012). Evaluating the energy and carbon reductions resulting from resource-efficient household stock. Energy and Buildings, 55, 422-432.

Beal, C. D., Gurung, T. R., \& Stewart, R. A. (2016). Demand-side management for supply-side efficiency: Modeling tailored strategies for reducing peak residential water demand. Sustainable Production and

Cominola, A., Giuliani, M., Piga, D., Castelletti, A., Rizzoli, A. (2017a) A Hybrid Signature-based Iterative Disaggregation algorithm for Non-Intrusive Load Monitoring. Applied Energy, 185, 331-344

Escriva-Bou, A., Lund, J.R., Pulido-Velazquez, M., 2015a. Modeling residential water and related energy, carbon footprint and costs in california. Environ. Sci. Policy 50, $270 \mathrm{e} 281$.

Giurco, D., Carrard, N., McFallan, S., Nalbantoglu, M., Inman, M., Thornton, N. \& White, S. (2008a). Residential end-use measurement guidebook: a guide to study design, sampling and technology. Prepared by the Institute for Sustainable Futures, UTS and CSIRO for the Smart Water Fund, Victoria.

Mayer, P.W., DeOreo, W.B., 1999. Residential End Uses of Water. American Water Works Association.

Nguyen, K.A., Zhang, H., and Stewart, R.A. (2013). Intelligent pattern recognition model to automate the categorisation of residential water end-use events. Journal of Environmental Modelling and Software, 47, 108-127.

Nguyen, K.A., Zhang, H., Stewart, R.A. (2011). Application of Dynamic Time Warping algorithm in prototype selection for the disaggregation of domestic water flow data into end use events. Proceeding of the 34th World Congress of the International Association for Hydro-Environment Engineering and Research, Brisbane, Australia, 26 June-1 July, 2011.

Nguyen, K.A., Stewart, R.A. and Zhang H. (2014). An autonomous and intelligent expert system for residential water end-use classification. Journal of Expert Systems with Application, 41(2), 342-356.

Nguyen, K.A., Stewart, R.A. and Zhang H. (2015). Intelligent autonomous system for water end-use classification: Autoflow. Applied Soft Computing, 31, 118-131.

Neenan B, Hemphill RC. Societal benefits of smart metering investments. Electr J 2008;21, 32-45.

Zoha, A., Gluhak, A., Imran., M.A., Rajasegarar, S (2012) Non-Intrusive Load Monitoring Approaches for Disaggregated Energy Sensing: A Survey. Sensors, 12(12), 16838-16866. 